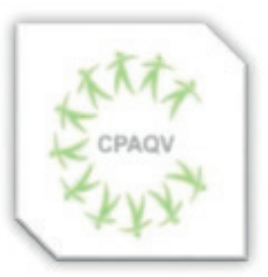

ISSN: 2178-7514
ARTIGO ORIGINAL

ATIVIDADE FÍSICA, CONSUMO ALIMENTAR E QUALIDADE DE VIDA DE PROFISSIONAIS DE SAÚDE EM HOSPITAIS

Physical activity, food consumption and quality of life of health professionals in hospital

Cristina Maria Mendes Resende ${ }^{1}$, Luane Pricila Cavalcante de Freitas ${ }^{2}$, Andréia Sousa de Silveira ${ }^{2}$, Edjane Freitas Silva ${ }^{2}$, Fabrício Olinda de Souza ${ }^{3}$, Eduardo Eriko Tenório ${ }^{4}$, Ricardo Freitas Dias ${ }^{1}$, Emília Chagas Costa ${ }^{5}$, Fabrício Cieslak ${ }^{6}$, Marco Aurélio de Valois Correia Junior ${ }^{1,7}$

Vol. 13 | No. 2| Ano 2021

\title{
RESUMO
}

Objetivo: Comparar a qualidade de vida, o consumo alimentar e o nível de atividade física entre diferentes profissionais de saúde que trabalhavam em unidades de terapia intensiva (UTIs) e enfermarias. Métodos: Estudo transversal e analítico realizado em UTI's e enfermarias adultos. A qualidade de vida foi avaliada pelo word health organization quality of life instrument bref (WHOQOL - bref), o consumo alimentar pelo Sistema de Vigilância Alimentar e Nutricional (SISVAN) e o nível de atividade física pelo international physical activity questionnaire (IPAQ - versão curta). Resultados: Foram avaliados 252 profissionais de saúde. Trabalhadores da enfermaria apresentaram melhor qualidade de vida do que a UTI nos domínios físico $(4,12 \pm 0,51$ vs $3,94 \pm 0,48 ; p=0,006)$ e psicológico $(4,06 \pm 0,51$ vs $3,86 \pm 0,46 ; \mathrm{p}=0,002)$. Quase metade dos indivíduos foram classificados como inativos, nã̃o apresentaram hábitos diários relacionada alimentação saudável e apresentaram práticas alimentares pouco recomendadas mais do que duas vezes por semana. Conclusões: A enfermaria apresentou melhor qualidade de vida do que a UTI. A formação na área da saúde deveria estimular hábitos de vida saudáveis devido um maior conhecimento sobre a gênese e fisiopatologia das doenças, no entanto quase a metade da população estudada estavam inativos e não apresentavam hábitos saudáveis relacionados a alimentação.

Palavras-chave: Atividade física; Consumo alimentar; Qualidade de vida; Profissionais de saúde

\begin{abstract}
Objective: To compare quality of life, food consumption, and level of physical activity among different health professionals working in intensive care units (ICUs) and wards. Methods: Cross-sectional and analytical study carried out in ICUs and adult wards. Quality of life was assessed by the Word Health Organization Quality of life instrument (WHOQOL - bref), food consumption by the Food and Nutrition Surveillance System (SISVAN) and the level of physical activity by the International Physical Activity questionnaire (IPAQ - short version). Results: 252 health professionals were evaluated. Nursing workers presented better quality of life than the ICU in the physical (4.12 \pm 0.51 vs $3.94 \pm 0.48 ; p=0.006)$ and psychological $(4.06 \pm 0.51$ vs $3.86 \pm 0.46 ; p=0.002)$. Almost half of the individuals were classified as inactive, had no daily habits related to healthy eating and presented poorly recommended dietary practices more than twice a week. Conclusion: The ward presented a better quality of life than the ICU. Health education should stimulate healthy life habits due to a greater knowledge about the genesis and pathophysiology of diseases, however, almost half of the studied population were inactive and had no healthy eating habits.
\end{abstract}

Keywords: Physical Activity, Food Consumption, Quality of Life, Health Professionals

\footnotetext{
${ }^{1}$ Universidade de Pernambuco, Programa de Pós-Graduação em Hebiatria, Faculdade de Odontologia, Recife, PE, Brasil 2Universidade de Pernambuco, Programa de Iniciação Científica, Departamento de Fisioterapia, Petrolina, PE, Brasil. 3Universidade Federal do Vale do São Francisco, Gestão Hospitalar, Petrolina, PE, Brasil. 4Universidade Federal da Paraíba, Departamento de Fisioterapia, João Pessoa, PB, Brasil. 5Universidade Federal de Pernambuco, Departamento de Educação Física, Recife, PE, Brasil. 6Universidade Federal do Paraná, Departamento de Educação Física, Curitiba, PR, Brasil.

7Universidade de Pernambuco, Programa de Pós-Graduação em Educação Física, Recife, PE, Brasil.
}

\section{Autor de correspondência}

Marco Aurélio de Valois Correia Junior/email: marcovalois@gmail.com 


\section{INTRODUÇÃO}

No ambiente hospitalar os profissionais de saúde estão expostos a uma série de riscos sejam eles físicos, químicos, mecânicos, biológicos e/ou psicológicos ${ }^{(1,2)}$. Além disso, o regime de turnos e plantões aliados a baixa remuneração e a necessidade de melhorar a renda familiar, faz com que muitos profissionais trabalhem em dois ou três empregos e tenham longas jornadas de trabalho, fatores esses que geram desgastes físicos, emocionais e afetam a qualidade do serviço prestado $^{(1,3,4)}$. Esses profissionais atuam diariamente com situações estressantes, que somadas a uma má alimentação, consumo de álcool, tabagismo e falta de tempo para a atividade física para o lazer e para o convívio familiar podem afetar a saúde e impactar negativamente no trabalho e na qualidade de vida ${ }^{(5-7)}$. Aliar um estilo de vida saudável, a uma alimentação adequada e a prática regular de atividade física é uma boa estratégia para melhorar a qualidade de vida ${ }^{(5,8)}$.

Escolhas alimentares saudáveis

são consideradas requisitos básicos para a promoção e proteção da saúde, além de auxiliar na menor incidência de doenças crônicas não transmissíveis ${ }^{(9,10)}$. A atividade física é outro fator importante que contribui para a saúde e promove o aumento da sensação de bem-estar, a qual oferece benefícios como recuperação da auto estima, redução do risco de doenças, diminuição da ansiedade e do estresse, sendo também uma forma de lazer e de restaurar o corpo das implicações danosas causadas por uma rotina de trabalho estressante $\mathrm{e}^{(11,12)}$.

Vários estudos avaliaram o nível de atividade física (NAF) e a qualidade de vida em diferentes populações ${ }^{(8,13,14)}$, no entanto poucas pesquisas investigaram o consumo alimentar ${ }^{(7)}$, a atividade física ${ }^{(4,11)}$ e a qualidade de vida ${ }^{(15,16)}$ nos profissionais que trabalharam em diferentes setores hospitalares ${ }^{(3,7,15,17,18)}$.

Com isso, observa-se a necessidade de pesquisar a saúde geral dos profissionais de saúde considerando os diversos setores do ambiente hospitalar, com o objetivo de identificar possíveis fatores de risco em potencial que a vida laboral impõe, e assim conscientizar e incentivar as pessoas a adotarem hábitos de vidas saudáveis em busca de uma melhor qualidade de vida ${ }^{(11)}$. Nesse sentido, os objetivos deste trabalho foram avaliar e comparar a qualidade de vida, o consumo alimentar e o nível de atividade física entre os profissionais de Unidade de Terapia Intensiva (UTI's) e enfermaria. Assim como, comparar a qualidade de vida, o consumo alimentar e o nível de atividade física entre diferentes profissionais de saúde que trabalhavam em UTI's e enfermarias.

\section{MATERIAIS E MÉTODOS}

Trata-se de um estudo transversal e analítico realizado com profissionais de saúde que trabalham em UTIs e enfermarias de atendimento à população adulta, nas cidades de Petrolina e Juazeiro. Essas cidades estão localizadas no Submédio São Francisco, na região do sertão brasileiro e possuem 
cerca de 491.927 habitantes ${ }^{(19)}$. Por meio do Departamento de Informática do Sistema Único de Saúde (DATASUS) foi realizada uma busca para identificar os hospitais da região que tinham o setor de Unidade de Terapia Intensiva adulto ${ }^{(20)}$. O período do estudo foi de fevereiro a dezembro de 2015. Foram incluídos na pesquisa os seguintes profissionais da área de saúde: médicos, enfermeiros, fisioterapeutas e técnicos de enfermagem, acima de 18 anos, de ambos os sexos, que possuírem vínculo empregatício com o hospital, não foram incluídos os colaboradores que durante o período de coleta estavam de licença maternidade, homens e mulheres que estiveram de férias ou licença médica.

Os dados pessoais foram coletados a partir de um formulário, contendo quesitos sobre nome, idade, sexo, categoria profissional, carga horária de trabalho, se possuía outro vínculo empregatício, tempo de serviço, renda salarial mensal, tabagismo, consumo de bebida alcoólica, massa corporal e estatura. A massa corporal dos indivíduos foi analisada pelo uso da balança digital (G-TECH ${ }^{\circledR}$, Pernambuco,Brasil) e a estatura foi mensurada utilizando uma fita métrica (Jomarca ${ }^{\circledR}$, São Paulo, Brasil).

O Índice de Massa Corpórea (IMC) foi calculado de acordo com a fórmula padrão IMC $=$ Peso $(\mathrm{kg}) /$ altura $\left(\mathrm{m}^{2}\right)$. O estado nutricional dos participantes foi classificado com base nos pontos de corte propostos pela World Health Organization ${ }^{(21)}$ : Eutrofia (IMC entre 18,5 e 24,9 kg/m²), sobrepeso (IMC entre 25 e $29,9 \mathrm{~kg} / \mathrm{m}^{2}$ ) e obesidade (IMC > $30 \mathrm{~kg} / \mathrm{m}^{2}$ )

Os questionários para avaliar prevalência de tabagismo, consumo de álcool, nível de atividade física, consumo alimentar e qualidade de vida foram aplicados em ambiente reservado, individualmente e em forma de entrevista com dois pesquisadores do programa de fortalecimento acadêmico da Universidade de Pernambuco (PFA/UPE) e o tempo gasto não ultrapassou 30 minutos.

A prevalência do tabagismo foi caracterizada pela resposta sim a pergunta "Você fuma? Se sim, quantos cigarros por dia?” e o consumo de álcool foi avaliado com a pergunta "Costuma fazer uso de bebida alcoólica?”.

Para avaliar o nível de atividade física (NAF) foi utilizado o questionário internacional de atividade física (IPAQ) em sua versão curta traduzida e validada para o Brasil(2). As perguntas foram relacionadas ao tempo gasto realizando atividade física na última semana, incluíram as atividades que fez no trabalho, para ir de um lugar a outro e para lazer e esporte. A análise dos resultados é feita seguindo os critérios de frequência, duração e intensidade, classificando o individuo em muito ativo, ativo, irregularmente ativo ou sedentário.

Foram considerados ativos os indivíduos classificados no IPAQ em ativo e muito ativo, onde para isso foi preciso atingir os seguintes critérios: a) atividade física vigorosa com frequência igual ou superior a três dias/semana com duração igual ou maior que 20 minutos/sessão; b) atividade física moderada ou caminhada com frequência igual ou superior a cinco dias/semana e duração igual ou maior que 30 minutos/sessão; c) qualquer atividade física cuja frequência somada fosse igual ou superior a cinco dias/semana e com duração igual ou maior que 150 minutos/semana. Os sujeitos que não alcançaram os critérios supracitados (irregularmente ativos e sedentários segundo o IPAQ) foram classificados como inativos. 
O consumo alimentar foi avaliado por meio do Formulário de Marcadores do Consumo Alimentar para Indivíduos Maiores de cinco anos de idade, que consta no Protocolo do Sistema de Vigilância Alimentar e Nutricionais SISVAN $^{(23)}$. Instrumento este que busca retratar a ingestão habitual de alimentos, trata-se de registro retrospectivo da frequência de consumo de alguns alimentos e bebidas nos últimos sete dias. O mesmo está relacionado tanto a uma alimentação saudável (exemplo: consumo diário de feijão, frutas, verduras) como a práticas pouco recomendadas (exemplo: consumo frequente de alimentos fritos e guloseimas) ${ }^{(23)}$, foi considerado consumo frequente, quando este era maior que duas vezes por semana. Esse formulário visa caracterizar de forma ampla o padrão alimentar do indivíduo e não pretende quantificar a dieta em termos de calorias e nutrientes, mas sim indicar a qualidade da alimentação em suas características tanto positivas como negativas ${ }^{(25)}$.

A Qualidade de Vida foi avaliada por meio do questionário Word Health Organization Quality of Life Instrument Bref (WHOQOLBref), traduzido e validado pelo grupo de estudos em qualidade de vida da Organização Mundial de Saúde (OMS) no Brasil, é um instrumento de fácil aplicação e compreensão para indivíduos adulto ${ }^{(24)}$. O WHOQOL-Bref é uma versão reduzida do World Health Organization Quality of Life Instrument 100 (WHOQOL-100), composto de 26 questões, sendo duas delas gerais de qualidade de vida. Esse instrumento é composto por quatro domínios da qualidade de vida, cada domínio tem por objetivo analisar, a capacidade física, o bem estar psicológico, as relações sociais e o meio ambiente onde o indivíduo está inserido, sendo composto também por um domínio que analisa a qualidade de vida global. Cada domínio é composto por questões, cujas pontuações das respostas variam entre um e cinco. Os escores finais de cada domínio são calculados por uma sintaxe, que considera as respostas de cada questão que compõe o domínio, resultando em escores finais numa escala likert.

Os dados foram analisados com auxílio dos softwares Statistical Package of Social Sciences (SPSS) 20.0 e o GraphPad Prism 5. Uma análise descritiva das variáveis foi primeiramente conduzida. Para testar a suposição de normalidade dos dados foi realizado o teste de Shapiro-Wilk. Para a avaliação das diferenças entre as proporções foram utilizados os teste de Qui-quadrado e Exato de Fisher. A comparação entre os grupos foi realizada por meio do One Way Anova com pósteste de Tukey e teste $\mathrm{t}$ de Student para variáveis paramétricas ou o teste de Kruskall-Wallis com pósteste de Dunn para as variáveis não paramétricas. As conclusões foram todas tomadas adotando-se um nível de significância de 5\%.

Esta pesquisa foi aprovada pelo Comitê de Ética em Pesquisa da Universidade de Pernambuco em 06 de maio de 2015 ( ${ }^{\circ}$ 1.054.035). Foram incluídos no estudo somente os indivíduos que aceitaram participar da pesquisa e assinaram o Termo de Consentimento Livre e Esclarecido (TCLE). 


\section{RESULTADOS}

A Figura 1 mostra o fluxograma dos hospitais que participaram do estudo, buscouse avaliar todos os hospitais que possuíam UTI's clínica adulto na região estudada. Foram encontrados sete hospitais com UTI's clínica adulto, sendo duas instituições civis filantrópicas e cinco particulares. Destes quatro hospitais se recusaram a participar da pesquisa, sendo incluídos neste trabalho os três que aceitaram. Participaram da pesquisa 252 profissionais saúde (13 médicos, 19 fisioterapeutas, 63 enfermeiros, 157 técnicos), desses 161 trabalhavam nas enfermarias (42 enfermeiros, 6 fisioterapeutas, 1 médico e 112 técnicos de enfermagem) e 91 nas UTI's (21 enfermeiros, 13 fisioterapeutas, 12 médicos e 45 técnicos de enfermagem).

Figura1.Fluxo de entradados profissionais do estudo de saúde que trabalham em UTIs e enfermarias de atendimento à população adulta, nas cidades de Petrolina e Juazeiro, no período de fevereiro a dezembro de 2015. UTI = Unidade de Terapia Intensiva.

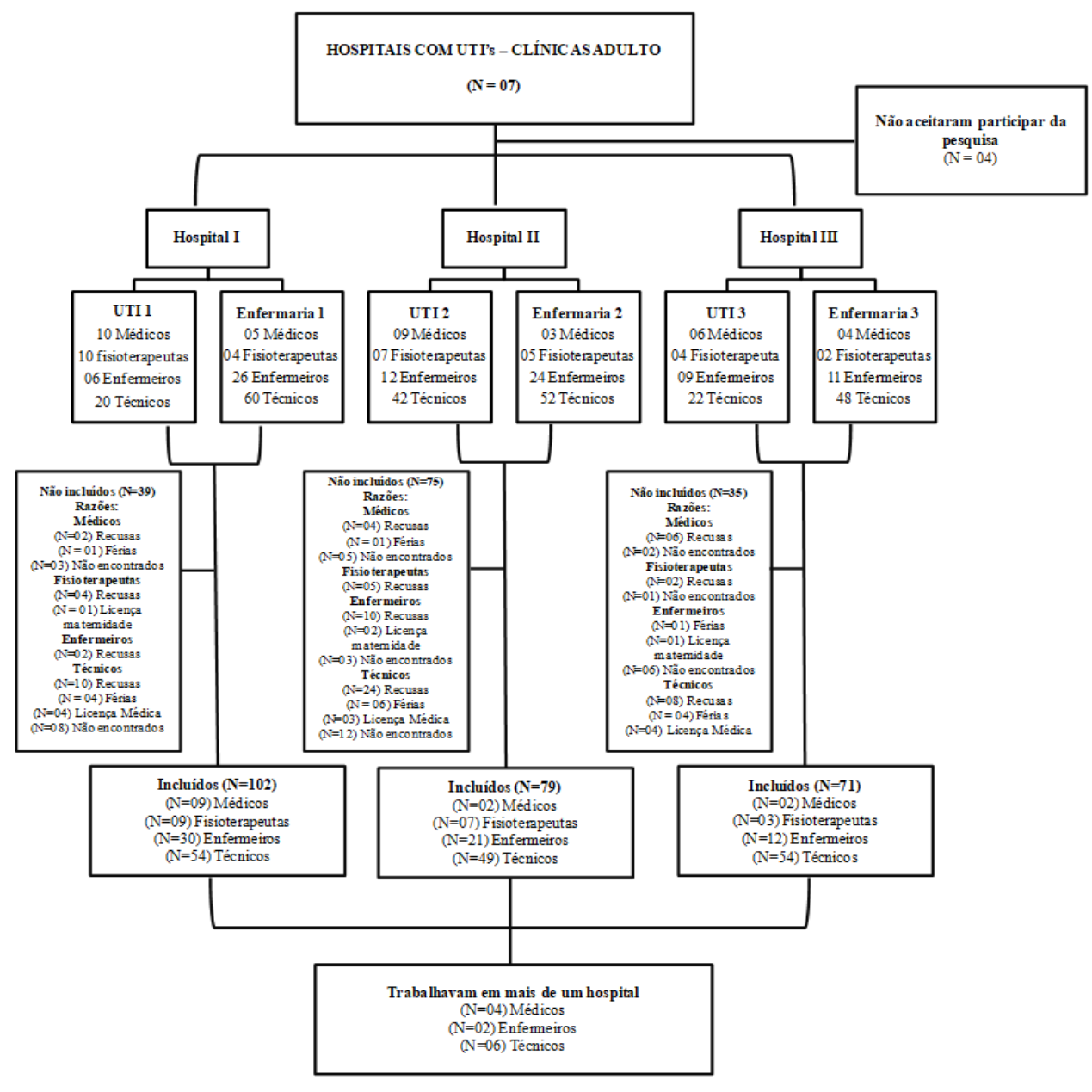


A comparação das variáveis idade, peso, altura, IMC, jornada, tempo de trabalho, gênero, domínios da qualidade de vida, tabagismo, etilismo, número de vínculos empregatícios, renda e atividade física entre profissionais de saúde que trabalham em UTI's e enfermarias para adultos estão apresentados na Tabela 1. Nas enfermarias as idades foram maiores, o sexo feminino foi mais prevalente e os domínio físico e psicológico da qualidade de vida apresentaram maiores escores. Com relação a renda salarial a maior parte dos indivíduos ganhavam menos do que $\mathrm{R} \$ 5.000,00$, com grande diferença entre a prevalência de profissionais que ganhavam mais que $\mathrm{R} \$ 10.000,00$. É importante destacar que $46 \%$ dos funcionários das UTI's e 48\% dos funcionários das Enfermarias foram inativos.

Tabela 1. Comparação entre idade, medidas antropométricas, jornada e tempo de trabalho, gênero, domínio de qualidade de vida, tabagismo, etilismo, número de vínculos empregatício, atividade física e consumo alimentar entre profissionais de saúde em UTI's e Enfermarias.

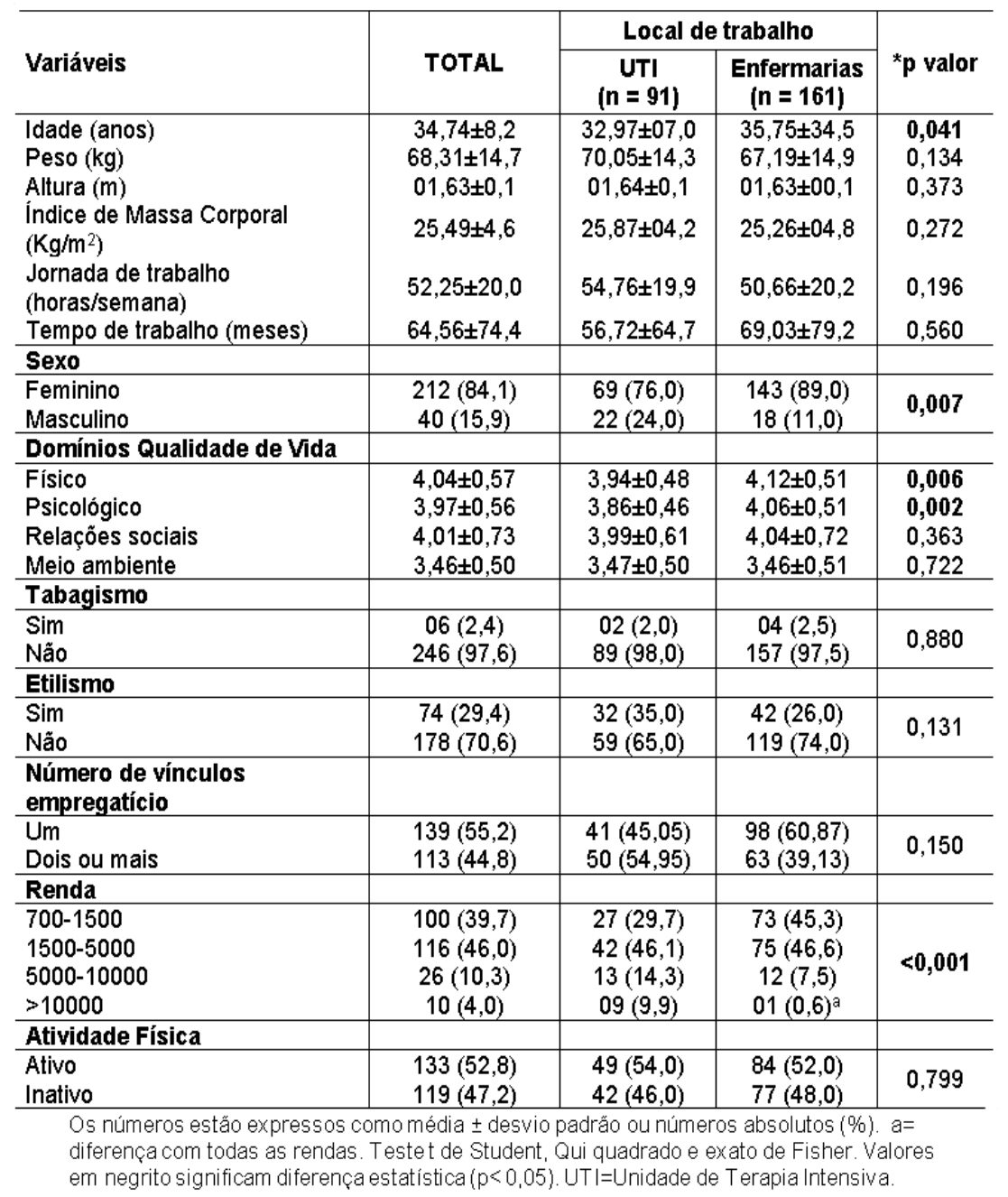


A Tabela 2 demonstra que não houve diferença no consumo entre os funcionários que trabalham em UTI e enfermarias, tanto para os alimentos ou bebidas relacionados a uma alimentação saudável quanto às práticas alimentares pouco recomendadas. É importante destacar que independente do local de trabalho, menos de 50\% dos indivíduos não apresentaram o hábito diário de consumir alimentos relacionados a uma alimentação saudável como salada crua, legumes e verduras cozidos, frutas frescas e saladas de frutas, leite e iogurte e aproximadamente $50 \%$ dos indivíduos consomem bolachas/biscoitos salgados ou salgadinhos de pacotes mais de duas vezes por semana, prática pouco recomendada.

Tabela 2. Comparação do consumo alimentar entre profissionais de saúde em UTI's e Enfermarias.

\begin{tabular}{|c|c|c|c|c|}
\hline \multirow{3}{*}{ Consumo Alimentar } & \multicolumn{4}{|c|}{ Local de trabalho } \\
\hline & \multicolumn{2}{|r|}{ UTI } & \multicolumn{2}{|c|}{ Enfermaria } \\
\hline & $\mathbf{n}$ & $\%(\mathrm{IC}-95 \%)$ & $\mathbf{n}$ & $\%(I C-95 \%)$ \\
\hline \multicolumn{5}{|l|}{ Consumo diário de alimentos $^{*}$} \\
\hline Salada crua & 22 & $24,2(16-34)$ & 56 & $34,8(28-42)$ \\
\hline Legumes e verdu ras cozidos & 12 & $13,2(07-22)$ & 34 & $21.1(15-28)$ \\
\hline Frutas frescas e salada de frutas & 32 & $35,2(26-45)$ & 61 & $37,9(31-46)$ \\
\hline Feijāo & 45 & $49,5(39-59)$ & 100 & $62.1(54-69)$ \\
\hline Leite e iogurte & 32 & $35,2(26-45)$ & 69 & $42,9(35-51)$ \\
\hline \multicolumn{5}{|l|}{ Práticas pouco recomendadas ${ }^{\star \star}$} \\
\hline $\begin{array}{l}\text { Batata frita, batata de pacote e } \\
\text { salgadinhos fritos (coxinha, quibe, pastel } \\
\text { e etc) }\end{array}$ & 10 & $11(9-19)$ & 15 & $9,2(5-15)$ \\
\hline Hambúrguer e embutidos & 19 & $20,9(14-30)$ & 26 & $16,2(11-23)$ \\
\hline $\begin{array}{l}\text { Bolachasibiscoitos salgados ou } \\
\text { salgadinho de pacote }\end{array}$ & 38 & $41,8(32-52)$ & 77 & $47.8(40-55)$ \\
\hline $\begin{array}{l}\text { Bolachasibiscoitos doces ou recheados, } \\
\text { doces, balas e chocolates }\end{array}$ & 26 & $28,6(20-39)$ & 47 & $29,3(23-37)$ \\
\hline $\begin{array}{l}\text { Refrigerantes (não considerar diet ou } \\
\text { light) }\end{array}$ & 25 & $27.5(19-37)$ & 51 & $31,7(25-39)$ \\
\hline
\end{tabular}


A Tabela 3 demonstra as análises separadas por local de trabalho refere à idade, IMC, jornada de trabalho, gênero, atividade física, tabagismo, etilismo, número de vínculos empregatícios e domínios de qualidade de vida entre médicos, fisioterapeutas, enfermeiros e técnicos. Nas UTI's, foi observado que os técnicos apresentaram IMC maior que os fisioterapeutas e os enfermeiros, os fisioterapeutas apresentaram jornada de trabalho menor que os enfermeiros e técnicos. $\mathrm{O}$ sexo feminino foi menos prevalente no grupo dos médicos do que no grupo dos técnicos. Quando se avaliou os domínios de qualidade de vida meio ambiente, os técnicos apresentaram valores menores quando comparados aos médicos e fisioterapeutas. Em relação ao etilismo os técnicos apresentaram frequência menor que os médicos e enfermeiros. Nas enfermarias, os técnicos apresentaram maior idade do que os enfermeiros, maiores jornadas de trabalho do que fisioterapeutas e maior tempo de trabalho e maior qualidade de vida no domínio psicológico do que o grupo dos enfermeiros.
Em relação à renda (dados não demonstrado em tabela), foi observado que nas UTI's e enfermarias nenhum médico recebia valores menores que $R \$ 5.000,00$ e que a maior parte dos técnicos recebiam valores entre $R \$$ 700,00 e R \$ 1.500,00 (55,6\% nas UTI's e 64,3\% nas enfermarias) ou entre $\mathrm{R} \$ 1.500$ e $\mathrm{R} \$ 5.000$ (44,4\% nas UTI's e 34,8\% nas enfermarias). A maioria dos fisioterapeutas recebia entre $\mathrm{R} \$ 1.500$ e $\mathrm{R} \$ 5.000$ (84,6\% nas UTI's e 100\% nas enfermarias). Entre os enfermeiros a maioria dos funcionários recebiam entre $R$ \$ 1500 e $R \$ 5000$ (52,4\% nas UTI's e 69\% nas enfermarias), também foi observado uma prevalência alta entre enfermeiros que recebiam entre $\mathrm{R} \$ 5000$ e $\mathrm{R} \$ 10000$ (42,9\% nas UTI's e 28,6\% nas enfermarias).

Tabela 3. Caracterização e comparação das variáveis relacionadas à idade, IMC, jornada de trabalho, gênero, atividade física, tabagismo, etilismo, número de vínculos empregatícios e domínios de qualidade de vida entre médicos, fisioterapeutas, enfermeiros, técnicos em UTI's e enfermarias.

\begin{tabular}{|c|c|c|c|c|c|c|c|c|c|c|}
\hline \multirow[b]{2}{*}{ Variáveis } & \multicolumn{5}{|c|}{ UTI } & \multicolumn{5}{|c|}{ Enfermarias } \\
\hline & $\begin{array}{c}\text { Médicos } \\
(\mathbf{n}=12)\end{array}$ & $\begin{array}{c}\begin{array}{c}\text { Fisioterapeutas } \\
(\mathrm{n}=13)\end{array} \\
\end{array}$ & $\begin{array}{c}\text { Enfermeiros } \\
(\mathbf{n}=21)\end{array}$ & $\begin{array}{c}\text { Técnicos } \\
(\mathrm{n}=45)\end{array}$ & *p valor & $\begin{array}{c}\text { Médicos } \\
(\mathbf{n}=\mathbf{1})\end{array}$ & $\begin{array}{c}\text { Fisioterapeutas } \\
(n=6)\end{array}$ & $\begin{array}{c}\text { Enfermeir os } \\
(\mathbf{n}=\mathbf{4 2})\end{array}$ & $\begin{array}{l}\text { Técnicos } \\
(\mathbf{n}=112)\end{array}$ & "p valor \\
\hline Idade (anos) & $33,5(27-49)$ & $30,0(23-44)$ & $30,00(22-42)$ & $32,00(21-52)$ & 0,216 & 46 & $31(27-42)$ & $30,00(23-47)^{*}$ & $\begin{array}{c}36,50(21- \\
58)\end{array}$ & 0,000 \\
\hline $\mathrm{IMC}\left(\mathrm{kg} / \mathrm{m}^{2}\right)$ & $24,59 \pm 2,4$ & $23,99 \pm 4,09 *$ & $24,89 \pm 3,21^{*}$ & $27,18 \pm 4,63$ & 0,026 & 27,81 & $24,43(19-32)$ & $22,79(18-35)$ & $25,0(18-38)$ & 0,150 \\
\hline $\begin{array}{l}\text { Jornada de Trabalho } \\
\text { (h) }\end{array}$ & $60,00(24-68)$ & $30,00(30-60)$ & $60,00(36-80)^{* * *}$ & $\begin{array}{l}48,00(36- \\
131)^{* * *}\end{array}$ & 0,023 & $60)$ & $30(30-60)^{*}$ & $36,0(30-144)$ & $\begin{array}{l}38,00(30- \\
120)\end{array}$ & 0,049 \\
\hline $\begin{array}{l}\text { Tempo de trabalho } \\
\text { (meses) }\end{array}$ & $30(3-240)$ & $42(1-228)$ & $29,00(1-276)$ & $37,50(1-288)$ & 0,977 & 120 & $78,00(4-216)$ & $12,00(1-240)^{*}$ & $48,0(1-324)$ & 0,008 \\
\hline \multicolumn{11}{|c|}{ Sexo } \\
\hline $\begin{array}{l}\text { Feminino } \\
\text { Masculino }\end{array}$ & $\begin{array}{l}6(50 \%)^{*} \\
6(50 \%)\end{array}$ & $\begin{array}{l}10(76,9 \%) \\
3(23,1 \%)\end{array}$ & $\begin{array}{c}14(66,7 \%) \\
7(33,3 \%) \\
\end{array}$ & $\begin{array}{c}39(86,7 \%) \\
6(13,3 \%)\end{array}$ & 0,013 & $\begin{array}{c}0 \\
1(100 \%) \\
\end{array}$ & $\begin{array}{l}5(83,3 \%) \\
1(16,7 \%)\end{array}$ & $\begin{array}{l}37(88,1 \%) \\
05(11,9 \%) \\
\end{array}$ & $\begin{array}{c}101(90,2 \%) \\
11(9,8 \%)\end{array}$ & 0,120 \\
\hline \multicolumn{11}{|c|}{ Domínio de qualidade de vida } \\
\hline Físico & $4,21(3-4,57)$ & $4,14(3,28-4,57)$ & $\begin{array}{c}3,85(2,28- \\
4,71)\end{array}$ & $\begin{array}{c}4,00(2,85- \\
4,85)\end{array}$ & 0,566 & 4,85 & $4,21(3,42-4,42)$ & $\begin{array}{c}4,00(2,71- \\
5,00)\end{array}$ & $4,28(0-5,0)$ & 0,220 \\
\hline Psicológico & $\begin{array}{c}3,83(3,33- \\
4,66)\end{array}$ & $3,83(3,00-4,33)$ & $\begin{array}{c}3,83(2,50- \\
4,66)\end{array}$ & $\begin{array}{c}4,00(2,83- \\
4,66)\end{array}$ & 0,424 & 5,00 & $4,16(3,16-4,33)$ & $\begin{array}{c}4,00(2,66- \\
4,83)^{*}\end{array}$ & $4,16(0-6,66)$ & 0,027 \\
\hline Relaçốes sociais & $4(3,33-5,00)$ & $4,33(3,66-5,00)$ & $\begin{array}{c}4,00(2,00- \\
4,66)\end{array}$ & $\begin{array}{l}4,00(2,00- \\
5,00)\end{array}$ & 0,174 & 5,00 & $4,66(3,66-5,00)$ & $\begin{array}{c}4,00(1,66- \\
5,00)\end{array}$ & $4,00(0-5,00)$ & $0,016^{\dagger}$ \\
\hline Meio ambiente & $3,83 \pm 0,36^{*}$ & $3,72 \pm 0,44^{*}$ & $3,51 \pm 0,36$ & $3,28 \pm 0,52$ & 0,001 & 4,86 & $3,74(2,62-3,87)$ & $\begin{array}{c}3,56(2,75- \\
4,75)\end{array}$ & $\begin{array}{c}3,37(2,0- \\
4,75)\end{array}$ & $\mathbf{0 , 0 4 0 ^ { \dagger }}$ \\
\hline \multicolumn{11}{|c|}{ Tabagismo } \\
\hline $\begin{array}{l}\text { Sim } \\
\text { Não }\end{array}$ & $\begin{array}{c}- \\
12(100 \%)\end{array}$ & $\begin{array}{c}- \\
13(100 \%)\end{array}$ & $\begin{array}{c}1(4,8 \%) \\
20(95,2 \%)\end{array}$ & $\begin{array}{c}1(2,2 \%) \\
44(97,8 \%)\end{array}$ & 0,590 & $\begin{array}{c}0 \\
1(100 \%)\end{array}$ & $\begin{array}{c}0 \\
6(100 \%)\end{array}$ & $\begin{array}{c}01(2,4 \%) \\
41(97,6 \%)\end{array}$ & $\begin{array}{c}3(2,7 \%) \\
109(97,3 \%)\end{array}$ & 0,720 \\
\hline \multicolumn{11}{|c|}{ Etilismo } \\
\hline $\begin{array}{l}\text { Sim } \\
\text { Não }\end{array}$ & $\begin{array}{c}7(58,3 \%) \\
5(41,7 \%)^{*}\end{array}$ & $\begin{array}{l}4(30,8 \%) \\
9(69,2 \%)\end{array}$ & $\begin{array}{l}12(57,1 \%) \\
9(42,9 \%)^{*}\end{array}$ & $\begin{array}{c}9(20 \%) \\
36(80 \%)\end{array}$ & 0,016 & $\begin{array}{c}1(100 \%) \\
0\end{array}$ & $\begin{array}{c}0 \\
6(100 \%)\end{array}$ & $\begin{array}{l}10(23,8 \%) \\
32(76,2 \%)\end{array}$ & $\begin{array}{l}31(27,7 \%) \\
81(72,3 \%)\end{array}$ & 0,570 \\
\hline \multicolumn{11}{|c|}{ Número de vínculos empregatício } \\
\hline $\begin{array}{l}\text { Um } \\
\text { Dois ou mais }\end{array}$ & $\begin{array}{l}3(25 \%) \\
9(75 \%)\end{array}$ & $\begin{array}{l}7(53,8 \%) \\
6(46,2 \%)\end{array}$ & $\begin{array}{c}9(42,9 \%) \\
12(57,1 \%)\end{array}$ & $\begin{array}{l}22(48,9 \%) \\
23(51,1 \%)\end{array}$ & 0,290 & $\begin{array}{c}0 \\
1(100 \%)\end{array}$ & $\begin{array}{l}4(66,7 \%) \\
2(33,3 \%)\end{array}$ & $\begin{array}{l}25(59,5 \%) \\
17(40,5 \%)\end{array}$ & $\begin{array}{l}69(61,6 \%) \\
43(38,4 \%)\end{array}$ & 0,640 \\
\hline \multicolumn{11}{|c|}{ Atividade física } \\
\hline $\begin{array}{l}\text { Ativo } \\
\text { Inativo }\end{array}$ & $\begin{array}{l}8(66,7 \%) \\
4(33,3 \%)\end{array}$ & $\begin{array}{l}7(53,8 \%) \\
6(46,2 \%)\end{array}$ & $\begin{array}{l}11(52,4 \%) \\
10(47,6 \%)\end{array}$ & $\begin{array}{l}23(51,1 \%) \\
22(48,9 \%)\end{array}$ & 0,401 & $\begin{array}{c}1(100 \%) \\
0\end{array}$ & $\begin{array}{l}3(50 \%) \\
3(50 \%)\end{array}$ & $\begin{array}{c}22(52,4 \%) \\
20(47,6 \%)\end{array}$ & $\begin{array}{l}58(51,8 \%) \\
54(48,2 \%)\end{array}$ & 0,730 \\
\hline
\end{tabular}


A Tabela 4 demonstra o consumo tanto em relação à alimentação saudável, quanto alimentar entre médicos, fisioterapeutas, às práticas pouco recomendadas. Com exceção enfermeiros e técnicos sem considerarolocalde do consumo de feijão que foi mais prevalente trabalho. Não houve diferença entre o consumo no grupo dos técnicos.

Tabela 4. Caracterização e comparação das variáveis relacionadas o consumo alimentar entre médicos, fisioterapeutas, enfermeiros, técnicos

\begin{tabular}{|c|c|c|c|c|}
\hline Variáveis & $\begin{array}{c}\text { Médico } \\
(\mathrm{n}=13)\end{array}$ & $\begin{array}{c}\text { Fisioterapeuta } \\
(n=19)\end{array}$ & $\begin{array}{c}\text { Enfermeiro } \\
(n=63)\end{array}$ & $\begin{array}{l}\text { Técnico } \\
(\mathrm{n}=157)\end{array}$ \\
\hline \multicolumn{5}{|c|}{ Consumo diário de alimentos relacionados à alimentação saudável } \\
\hline Salada crua & $7(53,8 \% ; \mathrm{IC}=25-81)$ & $3(15,8 \% ; \mathrm{IC}=5-38)$ & $\begin{array}{c}16(25,4 \% ; \mathrm{IC}=16- \\
37)\end{array}$ & $52(33,1 \% ; \mathrm{IC}=26-41)$ \\
\hline Legumes e verduras cozidos & $5(38,5 \% ; \mathrm{IC}=18-65)$ & $3(15,8 \% ; \mathrm{IC}=5-38)$ & $\begin{array}{c}11(17,5 \% ; \mathrm{IC}=10- \\
29)\end{array}$ & $27(17,2 \% ; \mathrm{IC}=12-24)$ \\
\hline Frutas frescas e salada de frutas & $7(53,8 \% ; \mathrm{IC}=29-77)$ & $7(36,8 \% ; \mathrm{IC}=19-59)$ & $\begin{array}{c}16(25,4 \% ; \mathrm{IC}=16- \\
37)\end{array}$ & $63(40,1 \% ; \mathrm{IC}=33-48)$ \\
\hline Feijão & $3(23,1 \% ; \mathrm{IC}=7,5-51)$ & $7(36,8 \% ; \mathrm{IC}=19-59)$ & $\begin{array}{c}28(44,4 \% ; \mathrm{IC}=33- \\
57)\end{array}$ & $\begin{array}{c}107(68,2 \% ; \mathrm{IC}=60- \\
75)\end{array}$ \\
\hline Leite e iogurte & $2(15,4 \% ; \mathrm{IC}=3-43)$ & $6(31,6 \% ; \mathrm{IC}=15-54)$ & $\begin{array}{c}27(42,9 \% ; \mathrm{IC}=31- \\
55)\end{array}$ & $66(42 \% ; \mathrm{IC}=35-50)$ \\
\hline \multicolumn{5}{|c|}{ Práticas pouco recomendadas - frequência de consumo maior que duas vezes por semana } \\
\hline $\begin{array}{l}\text { Batata frita, batata de pacote e salgadinhos fritos (coxinha, } \\
\text { quibe, pastel e etc) }\end{array}$ & $\begin{array}{l}1(7,7 \% ; \mathrm{IC}=<0,01- \\
35)\end{array}$ & $\begin{array}{c}1(5,3 \% ; \mathrm{IC}=<0,01- \\
26)\end{array}$ & $\begin{array}{l}11(17,5 \% ; \mathrm{IC}=10- \\
29)\end{array}$ & $12(7,6 \% ; \mathrm{IC}=4-13)$ \\
\hline Hambúrguer e embutidos & $\begin{array}{c}1(7,7 \% ; \mathrm{IC}=<0,01- \\
35)\end{array}$ & $3(15,8 \% ; \mathrm{IC}=5-38)$ & $\begin{array}{l}16(25,4 \% ; \mathrm{IC}=16- \\
37)\end{array}$ & $\begin{array}{l}25(15,9 \% ; \mathrm{IC}=11- \\
22,5)\end{array}$ \\
\hline Bolachas/biscoitos salgados ou salgadinho de pacote & $5(38,5 \% ; \mathrm{IC}=18-65)$ & $7(36,8 \% ; \mathrm{IC}=19-59)$ & $\begin{array}{c}26(41,3 \% ; \mathrm{IC}=30- \\
54)\end{array}$ & $77(49 \% ; \mathrm{IC}=41-58)$ \\
\hline $\begin{array}{l}\text { Bolachas/biscoitos doces ou recheados, doces, balas e } \\
\text { chocolates }\end{array}$ & $5(38,5 \% ; \mathrm{IC}=18-65)$ & $6(31,6 \% ; \mathrm{IC}=15-54)$ & $\begin{array}{c}21(33,3 \% ; \mathrm{IC}=23- \\
46)\end{array}$ & $\begin{array}{l}41(26,1 \% ; \mathrm{IC}=20- \\
33,5)\end{array}$ \\
\hline Refrigerantes (não considerar diet ou light) & $\begin{array}{c}1(7,7 \% ; \mathrm{IC}=<0,01- \\
35)\end{array}$ & $4(21,1 \% ; \mathrm{IC}=8-44)$ & $\begin{array}{c}21(33,3 \% ; \mathrm{IC}=23- \\
46)\end{array}$ & $\begin{array}{c}50(31,8 \% ; \mathrm{IC}=25- \\
39,5)\end{array}$ \\
\hline
\end{tabular}

\section{DISCUSSÃO}

Esta pesquisa identificou que os profissionais da enfermaria apresentaram melhor qualidade de vida, idade mais avançada e o sexo feminino foi mais prevalente em comparação aos da UTI. Boa parte dos profissionais excediam uma jornada de trabalho de 40 horas e quase metade foram classificados como inativos. Independente do local de trabalho, menos de 50\% dos funcionários não apresentaram hábitos diários de consumir grupos de alimentos relacionados à alimentação saudável e aproximadamente 50\% dos indivíduos consumiam mais do que duas vezes por semana alimentos considerados como pouco recomendados.
Por ser um ambiente de alta complexidade os profissionais da UTI estão mais expostos à sobrecarga de funções e ambiente de estresse, o que torna os trabalhadores fisicamente mais desgastados ${ }^{(15)}$. Associado a essa problemática, eles necessitam trabalhar em estado de atenção total, lidarem com o sofrimento e até mesmo morte de seus pacientes de forma mais frequente do que em outras áreas do hospital. Isto pode ser uma das causas da menor qualidade de vida encontrada no domínio físico e psicológico ${ }^{(25)}$. Nesse contexto, é possível que a procura por uma melhor QV no ambiente laboral possa ter estimulado os profissionais da UTI a solicitarem transferência e realocação para outros setores 
do hospital deixando os sujeitos mais jovens e provavelmente menos tempo de trabalho nesse local.

A associação de um estilo de vida saudável, junto com a prática regular de atividade física, controle do tabagismo, etilismo e alimentação saudável melhoram a qualidade de vida e reduzem cerca de $50 \%$ a chance de desenvolver doenças crônicas não transmissíveis (DCNT) (4,26,27). Chama atenção que quase a metade da população estudada era inativa, com idade menor que 40 anos e não apresentavam bons hábitos alimentares. Diante desse preocupante cenário é necessário que a gestão hospitalar lance estratégias de curto e longo prazo para a promoção da saúde do trabalhador tanto os da UTI quanto os da enfermaria.

A dieta da população brasileira ainda é baseada em alimentos tradicionais, como o arroz e feijão, porém há crescente aumento do consumo de sucos artificiais, refrigerantes e alimentos ricos em gorduras aliados ao baixo consumo de frutas, verduras e legumes ${ }^{(28)}$. Esses achados são importantes, pois a transição do consumo observado na população brasileira, se reflete até mesmo em profissionais de saúde no qual esperava-se uma alimentação de melhor qualidade. Neste trabalho, observou-se baixo consumo diário de frutas $(\sim 37 \%)$, salada crua $(\sim 30 \%)$, legumes e verduras $(\sim 20 \%)$, feijão $(\sim 50 \%)$ e leite e derivados ( $\sim 40 \%)$. Por outro lado, no que se refere aos alimentos pouco recomendados, boa parte dos profissionais consumiam mais do que duas vezes por semana hambúrguer e embutidos ( 20\%), biscoitos salgados $(\sim 45 \%)$, bolachas doces $(\sim 29 \%)$ e refrigerantes $(\sim 30 \%)$.

Quando a terapia intensiva e a enfermaria foram avaliadas separadamente, os técnicos de enfermagem da UTI apresentaram maior IMC que os fisioterapeutas e enfermeiros, menores valores para domínio de qualidade de vida meio ambiente do que médicos e fisioterapeutas e menor frequência de etilismo do que médicos e enfermeiros. Nas enfermarias, os técnicos apresentaram maior idade do que os enfermeiros, maiores jornadas de trabalho do que fisioterapeutas e maior tempo de trabalho e maior qualidade de vida no domínio psicológico do que o grupo dos enfermeiros.

A diferença encontrada no domínio meio ambiente pode ser explicado devido às perguntas do questionário estarem voltadas á recursos financeiros, lazer, ambiente físico, transporte e lar, sendo que estes profissionais geralmente trabalham em mais de um turno, além de terem uma renda salarial menor, que muitas vezes não supri suas necessidades ${ }^{(27,28)}$. Além disso, todos os técnicos de enfermagem apresentam renda inferior a $\mathrm{R} \$ 5.000,00$ mil reais e a maioria $(55,6 \%)$ recebem salários inferior a $\mathrm{R} \$ 1.500,00$ reais, diferentemente dos médicos que ganhavam um salário acima de $\mathrm{R} \$ 5000,00$ reais, e dos $(89,4 \%)$ fisioterapeutas (92,3\%) e enfermeiros (95,3\%) que ganhavam mais do que $\mathrm{R} \$ 1500,00$ reais.

Existe uma relação direta no que se refere as longas jornada de trabalho e a renda 
salarial, isso ocorre provavelmente com o intuito de complementar a renda familiar e adquirir estabilidade financeira o que impulsiona a busca por mais de um emprego e pode ter sido a razão da alta carga de trabalho encontrada nessa pesquisa $(52,25 \pm 20,0$ vs 44 horas por semana de acordo com as normas brasileiras) ${ }^{29}$. Associada ao aumento da carga horária, ocorre diminuição do tempo livre para a prática de atividade física, atividades de lazer e cuidados com a saúde ${ }^{(3,4)} \mathrm{O}$ que demonstra maior risco destes sujeitos em desenvolver doenças crônicas não transmissíveis.

Quando foi avaliado o consumo alimentar entre médicos, fisioterapeutas, enfermeiros e técnicos sem considerar o local de trabalho, só houve diferença do consumo de feijão que foi mais prevalente no grupo dos técnicos de enfermagem. De acordo com Coelho, Aguiar e Fernandes ${ }^{(30)}$, o aumento da renda tende a elevar o consumo domiciliar de produtos como queijos e carne bovina de primeira e diminuir o consumo de produtos básicos, como arroz e feijão, isso pode justificar o consumo maior de feijão no grupo dos técnicos pois é o grupo com menor renda dentre os estudados.

Uma possível limitação desse estudo é a validação externa da amostra, visto o caráter regional, no entanto tentou-se avaliar todos hospitais da região que tinham UTI's adulto em seu serviço. Uma outra questão foi o pouco tempo disponível para a resposta dos questionários por parte dos profissionais, pois foram realizados em âmbito hospitalar, durante a rotina de trabalho, algumas vezes as entrevistas precisavam ser interrompidas. Outras questões importantes foram à recusa em responder as perguntas, principalmente os médicos e bem como falta de uma avaliação mais aprofundada do consumo alimentar, visto que os questionários deveriam ser curtos para facilitar a aceitação e resposta por parte dos profissionais. Apesar disso, destaca-se a importância desse estudo, como parâmetros para direcionar o desenvolvimento de estratégias que respondam às necessidades e promovam à saúde, com o objetivo de diminuir os efeitos negativos causados pelas altas jornadas de trabalho, rotina estressante do ambiente e consumo alimentar inadequado. Destaca-se a necessidades de estratégias para o incentivo a adoção de hábitos alimentares saudáveis e a prática regular de atividade física.

\section{CONCLUSÃO}

Conclui-se que a enfermaria apresentou melhor qualidade de vida e idade mais avançada em comparação aos da UTI. Houve um excesso da jornada de trabalho e quase metade do participantes foram classificados como inativos. Menos de 50\% dos funcionários não apresentaram hábitos diários de consumir grupos de alimentos relacionados à alimentação saudável e aproximadamente metade dos indivíduos consumiam mais do que duas vezes por semana alimentos considerados como pouco recomendados. 


\section{REFERENCIAS}

1. SANTANA VS, FEITOSA AG, GUEDES LBA, SALES NBB. Qualidade de vida dos profissionais de saúde em ambiente hospitalar. Rev Pesquisa em Fisioterapia 2014; 4 (1): 35-4.

2. BEZERRA AMF, BEZERRA KKS, BEZERRA WKT, ATHAYDE ACR, VIEIRA AL. Riscos ocupacionais e acidentes de trabalho em profissionais de enfermagem no ambiente hospitalar. REBES 2015; 5 (2): 01-07.

3. ALVES EF. O significado de qualidade de vida para cuidadores de enfermagem em uma unidade de terapia intensiva adulto. Mundo Saúde 2013; 37 (4): 458-463.

4. LIMA DMG, ARAÚJO, RC, PITANGUI ACR, RIZZO JA, SARINHO SW; SANTOS CMA, et al. Descrição da atividade física e da jornada de trabalho na qualidade de vida de profissionais de terapia intensiva: Comparação entre um grande centro urbano e uma cidade do interior brasileiro. Rev Bras Ativ Fis Saúde 2015; 20 (4): 386-396.

5. ACIOLI NETO ACF, ARAÚJO RC, PITANGUI ACR, MENEZES LC, FRANÇA EET, COSTA, et al. Qualidade de vida e nível de atividade física de profissionais de saúde de unidades de terapia intensiva. Rev Bras Ativ Fis e Saúde 2013; 18 (6): 711-719.

6. FOGACA MC, CARVALHO WB, MARTINS LAN. Estudo preliminar sobre a qualidade de vida de médicos e enfermeiros intensivistas pediátricos e neonatais. Rev Esc Enferm 2010; 44 (3): 708-712.

7. FREIRE CB, DIAS RF, SCHWINGEL PA, FRANÇA EET, ANDRADE FMD, COSTA EC, et al. Qualidade de vida e atividade física em profissionais de terapia intensiva do sub médio São Francisco. Rev Bras Enferm 2015; 68 (1): 26-31.

8. PUCCI GCMF, RECH CR, FERMINO RC, REIS, RS. Associação entre atividade física e qualidade de vida em adultos. Rev Saúde Pública 2012; 46 (1): 166-179.

9. BUSATO MA, PEDROLO C, GALLINA LS, ROSA L. Ambiente e alimentação saudável: percepções e práticas de estudantes universitários. Semina: Ciências Biológicas e da Saúde 2015; 36 (2): 75-84.

10. NEVES ACM, GONZAGA LAA, MARTENS, IBG, MOURA, EC. Validação de indicadores do consumo de alimentos e bebidas obtidos por inquérito telefônico em Belém, Pará, Brasil. Cad. Saúde Pública 2010; 26 (12): 23792388.

11. SILVA RS, SILVA I, SILVA RA, SOUZA L, TOMASI E. Atividade física e qualidade de vida. Ciênc. Saúde Coletiva 2010; 15 (1): 115-120.

12. ROCHA SV, PIE ACS, CARDOSO JP, AMORIM CR, CARNEIRO LRV, VILELA ABA. Nível de atividade física entre funcionários de uma instituição de ensino superior da Bahia. Ulbra Mov 2011; 2 (1): 16-29.

13. MACEDO RM, OLIVEIRA MRP, CILIÃO MR, PROSDÓCIMO ACG, MACEDO ACB, FRANCA $D$, et al. Nível de atividade física de idosos participantes de um programa de prevenção de doença cardiovascular. ASSOBRAFIR Ciência 2015; 6 (3): 11-20.

14. MALTA DC, ANDRADE SSA, SANTOS M, RODRIGUES GBA, MIELKE GI. Tendências dos indicadores de atividade física em adultos: Conjunto de capitais do Brasil 2006-2013. Rev Bras Ativ Fís Saúde 2015; 20 (2): 141-151.

15. BORGES T; BIANCHIN MA. Qualidade de vida dos profissionais de enfermagem de um hospital universitário do interior de São Paulo. Arq. Ciênc. Saúde 2015; 22 (1): 55-58. 16. OLIVARES A, BONITO J, SILVA R. Qualidade de vida no trabalho dos médicos da atenção básica no estado de Roraima (Brasil). Rev Psicologia, Saúde e Doenças 2015; 16 (1): 100-111.

17. PRETTO ADB, PASTORE CA, ASSUNCÃO

MCF. Comportamentos relacionados à saúde entre profissionais de ambulatórios do Sistema Único de Saúde no município de Pelotas-RS. Epidemiol. Serv. Saúde 2014; 23 (4): 635-644.

18. MACIEL MED, OLIVEIRA FN. Qualidade de vida do profissional técnico de enfermagem: a realidade de um hospital filantrópico em Dourados-MS. Rev Psicologia e Saúde; 2014; 6 (1): 83-89.

19. INSTITUTO BRASILEIRO DE GEOGRAFIA E ESTATÍSTICA (IBGE). Censo Populacional do Brasil; 2010. [acessado em 25 de abril de 2016]. Disponível em: <www.censo2010.ibge.gov.br/sinopse>.

20. BRASIL, MINISTÉRIO DA SAÚDE (MS). BANCODE DADOS DO SISTEMA ÚNICO DE SAUUDEDATASUS; 2015. Disponível em http://www.datasus.gov.br [Acessado em 08 de maio de 2015].

21. WORLD HEALTH ORGANIZATION (WHO). Obesity: preventing and managing the global epidemic Obesity: preventing and managing the global epidemic; 2000, Report of a WHO Consultation (WHO Technical Report Series 894).

22. MATSUDO S, ARAÚJO T, MATSUDO V, ANDRADE D, ANDRADE E, OLIVEIRA C, et al. Questionário Internacional de Atividade Física (IPAQ): estudo de validade e reprodutibilidade no Brasil. Rev Bras Ativ Fís Saúde 2001; 6 (2): 5-12.

23. BRASIL. PROTOCOLOS DO SISTEMA DE VIGILÂNCIA ALIMENTAR ENUTRICIONAL-SISVAN NA ASSISTÊNCIA A SAÚDE. Secretaria da atenção a saúde. Departamento de atenção básica. Coordenação Geral da Política de Alimentação e Nutrição. Brasilia: Ministério da Saúde; 2008.

24. FLECK MPA, LOUZADA S, XAVIER M, CHACHAMOVICH E, VIEIRA G, SANTOS et al. Aplicação da versão em português do instrumento abreviado de avaliação da qualidade de vida "WHOQOL-bref". Rev Saúde Pública 2000; 34 (2): 178-183.

25. SALES JC, BORGES CM, ALVES OVM, PAES LW, CAMPOS ACV. Qualidade de vida de três categorias profissionais da saúde em um hospital de Minas Gerais, Brasil. Rev enferm UFPE 2010; 4 (3): 1365-1370.

26. AZEVEDO ECC, DIA FMRS, DINIZ AS, CABRAL PC. Consumo alimentar de risco e proteção para as doenças crônicas não transmissíveis e sua associação com a gordura corporal: um estudo com funcionários da área de saúde de uma universidade pública de Recife (PE), Brasil. Ciênc. Saúde Coletiva 2014; 19 (5): 1613-1622.

27. RIOS KA, BARABOSA DA, BELASCO AGS. Avaliação da qualidade de vida e depressão dos técnicos e auxiliares de enfermagem. Rev. Latinoam. Enferm 2010; 18 (3): 122-130.

28. BRASIL. MINISTÉRIO DA SAÚDE (MS). PESQUISA DE ORCAMENTOS FAMILIARES 20082009. Antropometria e Estado Nutricional de Crianças, Adolescentes e Adultos no Brasil. Rio de Janeiro, RJ: IBGE; 2010.130p.

29. PLANALTO. Planalto.gov.br [homepage]. CONSOLIDAÇÃO DAS LEIS DO TRABALHO. [acessado em 23 abr.2016]. Disponível em: < http://www.planalto.gov. br/ccivil_03/Decreto-Lei/Del5452. htm\#art478J2>.

30. COELHO AB, AGUIAR DRD, FERNANDES EA. Padrão de consumo de alimentos no Brasil. RESR 2009; 47 (2): 335-362.

OBSERVAÇÃO: Os autores declaram não existir conflitos de interesse de qualquer natureza. 